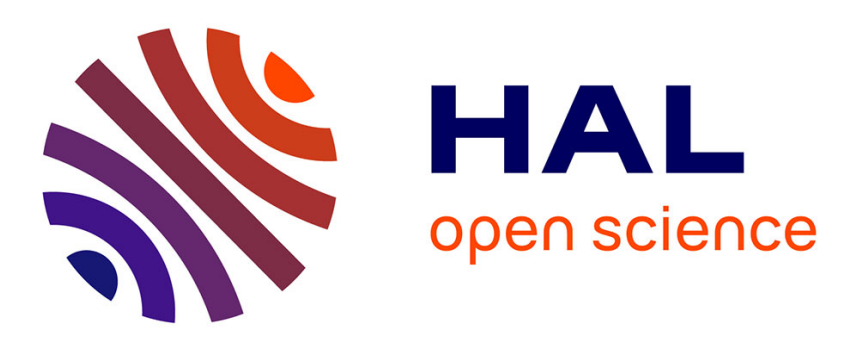

\title{
The modal substructuring method: An efficient technique for large-size numerical simulations
}

Pierre Olivier Laffay, Olivier Quéméner, Alain Neveu, Bilal Elhajjar

\section{To cite this version:}

Pierre Olivier Laffay, Olivier Quéméner, Alain Neveu, Bilal Elhajjar. The modal substructuring method: An efficient technique for large-size numerical simulations. Numerical Heat Transfer, Part B Fundamentals, 2011, 60 (4), pp.278-304. 10.1080/10407790.2011.609113 . hal-01176731

\section{HAL Id: hal-01176731 \\ https://hal.science/hal-01176731}

Submitted on 18 Jul 2018

HAL is a multi-disciplinary open access archive for the deposit and dissemination of scientific research documents, whether they are published or not. The documents may come from teaching and research institutions in France or abroad, or from public or private research centers.
L'archive ouverte pluridisciplinaire HAL, est destinée au dépôt et à la diffusion de documents scientifiques de niveau recherche, publiés ou non, émanant des établissements d'enseignement et de recherche français ou étrangers, des laboratoires publics ou privés. 


\title{
THE MODAL SUBSTRUCTURING METHOD: AN EFFICIENT TECHNIQUE FOR LARGE-SIZE NUMERICAL SIMULATIONS
}

\author{
P. O. Laffay, O. Quéméner, A. Neveu, and B. Elhaijar \\ Laboratoire de Mécanique et d'Energétique d'Evry, Courcouronnes, \\ Evry Cédex, France
}

\begin{abstract}
This article presents an extension of the branch Eigenmodes reduction method (BERM) to substructuring models. One of the method's interests lies in its capacity to build a reduced modal model for complex geometries which are characterized by very large matrix sizes. This article treats a 3-D nonlinear application. Such a substructuring reduced model permits us to obtain precise results over the whole domain, with an important gain of CPU time. In the case considered, a global reduced branch model can also be built. A comparison between the two reduced models shows the interest of the substructuring technique.
\end{abstract}

\section{INTRODUCTION}

This work concerns modal reduction methods for thermal conduction problems, which permits us to decrease the CPU time significantly but with good precision over the whole simulated domain. Among the different techniques, such as the classical thermal modes computation [1,2], the modal identification Method (MIM) [3, 4], and proper orthogonal decomposition (POD) [5, 6], a recent one is the branch eigenmodes reduction method (BERM), which uses particular eigenmodes called branch modes [7-9]. The origin of the branch modes is that the branch eigenvalues problem uses an unusual boundary condition that depends on the eigenvalue, therefore the branch modes possess the distinctive feature of forming a basis for nonlinear thermal problems in the domain and on the boundary. The projection of a thermal problem on a reduced basis resulting from such branch eigenmodes yields a reduced problem called the state model, where the unknowns are the excitation states of the modes. Since the modal superposition technique is inherently linear, the difficulty of the nonlinearity is treated in the state equations, via a nonlinear coupling between the excitation states of the modes. Solving this system permits rebuilding the thermal field over the whole domain.

Although it is successful, there are some limitations to this method. One limitation is in obtaining the branch basis, because this calculation is made on the whole domain; in the case of a very complex mesh corresponding to a realistically physical 


\section{NOMENCLATURE}

$\begin{array}{ll}c & \text { volumic capacity } \\ h & \text { convective exchange coefficient } \\ k & \text { thermal conductivity } \\ T C R & \text { thermal contact resistance } \\ T(M, t) & \text { temperature field } \\ V(M) & \text { branch eigenmode } \\ x & \text { excitation state } \\ z & \text { eigenvalue } \\ \varepsilon_{\max } & \text { maximum error }\end{array}$

$\begin{array}{ll}\varepsilon_{\text {mean }} & \text { averaged in space error } \\ \zeta & \text { Steklov number } \\ \varphi & \text { surface heat flux } \\ \pi & \text { volume heat source } \\ \tau & \text { time constant } \\ & \\ \text { Superscript } & \\ \sim & \text { reduced notation } \\ - & \text { substructuring reduced notation }\end{array}$

problem, the order of the discretized model is important, and the memory requirements can exceed the machine's capacity. Thus, in the case for which this method is particularly justified, it is difficult to use it!

For such configurations, we propose using a substructuring method, in which the domain is separated into several adjacent subdomains, and where a set of eigenmodes is computed for each subdomain. We obtain different states models which are linked to each other by internal boundary conditions, and which can be solved before rebuilding the thermal field.

Initially developed for mechanical problems [10-12], analogous techniques have been used for numerical thermal problems [13]. Concerning the modal reduction methods, the substructuring technique has also been tested: The MIM method [14], for which the modal formulation is used without eigenvectors computation, uses the substructuring technique to link the MIM formulation with another model. For methods for which eigenvectors are computed, some articles use a classical thermal basis limited to linear problems: El Biyaali [15] proposes a numerical approach corresponding to a new diagonalization of the coupling system. Another technique is modal synthesis [16], in which specific coupling modes are added to each local basis, yielding a new basis in the full domain. This work uses a temperature jump and flux jump function to control these jumps on the internal boundaries between the different subdomains.

Concerning the BERM method, a first use of the substructuring technique was made to resolve a problem with a thermal contact resistance [17]. Indeed, in a such configuration, a substructuring model is the only way to use a reduced model. The application was constituted of two subdomains in a 2-D configuration. The size of the different matrices obtained after spatial discretization was not very important. The substructuring method's principle leads to a double value of temperature on the internal boundaries between the different subdomains. Thus a temperature jump function appears for which the weight coefficient is naturally linked to the thermal contact resistance of the treated application. In this configuration the modal substructuring technique allows one to obtain an important gain of CPU time, with precise results.

Nevertheless, this method has never been used for a more classical problem in which there is no thermal contact resistance, but where the size of the discretized geometry is large. For such configurations, several questions have to be asked:

1. Is it possible to use this method for a problem without thermal contact resistance?

2. How then is one to choose the parameters of the temperature jump? 
3. When it is possible to compute a branch basis on the whole domain, and then use the classical global BERM method, is the substructuring method more efficient?

These are the questions we propose to answer in this article. After a first part, in which we review the technique used, a second part presents a 3-D nonlinear application, constituted of three subdomains, without thermal contact resistance.

\section{REVIEW OF THE BERM METHOD}

We consider a classical conduction problem on the domain $\Omega$, receiving a volume flux $\pi$ and characterized by the thermal conductivity $k$ and the volume capacity $c$. This domain is defined by two external boundaries: $\Gamma a$, which is adiabatic, and $\Gamma e$, characterized by a convective condition (exchange coefficient $h$ and ambient temperature $T_{e}$ ). All the parameters can be functions of temperature and are time-dependent. The equations are the following:

$$
\begin{gathered}
\forall M \in \Omega \quad c \frac{\partial T}{\partial t}=\operatorname{div}(k \vec{\nabla} T)+\pi \\
\forall M \in \Gamma e \quad k \vec{\nabla} T \cdot \vec{n}=h\left(T_{e}-T\right) \\
\forall M \in \Gamma a \quad \vec{\nabla} T \cdot \vec{n}=0 \\
\forall M \in \Omega, t=0 \quad T=T_{e}
\end{gathered}
$$

The usual weak formulation of such a problem is, with $f$ the test function,

$$
\int_{\Omega} c \frac{\partial T}{\partial t} f \partial \Omega=-\int_{\Omega} k \overrightarrow{\nabla f} \cdot \vec{\nabla} T \partial \Omega-\int_{\Gamma e} h T f \partial \Gamma+\int_{\Omega} \pi f \partial \Omega+\int_{\Gamma e} h T_{e} f \partial \Gamma
$$

The discrete formulation corresponding to this equation after the spatial discretization is presented in the Appendix, where appear the principal discrete mathematical relations used in this article.

If a basis $\left(z_{i}, V_{i}\right)$ of the space of the solution is known, it is possible to project the thermal field on this basis.

$$
T(M, t)=\sum_{j=1}^{\infty} x_{j}(t) V_{j}(M)
$$

Here $z_{i}$ is the eigenvalue and $V_{i}$ is the eigenvector for the ith element of the base, and $x_{i}$ is the time-dependent amplitude, called the excitation state.

From this modal formulation, it is possible to reduce the number of unknowns of the problem, by substituting in Eq. (6) for the infinite sum by a finite sum of predominant terms.

At this stage, we still have to:

1. Describe and calculate the base

2. Build the state equation 
3. Define a reduction method which selects the predominant modes, according to a criterion to be specified

\subsection{The Branch Basis}

Among the different possible bases, the branch basis is particularly adapted to the thermal problem characterized by nonlinear and time-dependent boundary conditions. This basis is defined by the following equations:

$$
\begin{gathered}
\forall M \in \Omega \quad k_{0} \Delta V_{i}=z_{i} c_{0} V_{i} \\
\forall M \in \Gamma e \quad k_{0} \vec{\nabla} V_{i} \cdot \vec{n}=z_{i} \zeta V_{i} \\
\forall M \in \Gamma a \quad k_{0} \vec{\nabla} V_{i} \cdot \vec{n}=0
\end{gathered}
$$

where $k_{0}$ and $c_{0}$ are constant values of the thermal conductivity and the volume capacity.

The originality of the problem is the Steklov boundary condition [Eq. (8)], which depends on the eigenvalue $z_{i}$, and which is independent of the boundary conditions of the real thermal problem. In this way, the branch modes form a basisfor any thermal problem, including those characterized by parameters that are functions of time or of temperature. This is not true of the "classical" modes, such as the Dirichlet basis (the eigenfunction value is set to zero on the boundary), or the Fourier basis (the ratio between the eigenfunction and its gradient on the boundary is fixed). $\zeta$ is the Steklov coefficient, which ensures that the eigenvalue $z_{i}$ has the same dimension in Eqs. (7) and (8). The last relation defining the problem [Eq. (9)] is a particular condition which imposes for the modes a null gradient for adiabatic boundaries.

Using the weak formulation, Eqs. (7)-(9) lead to

$$
\int_{\Omega} k_{0} \overrightarrow{\nabla f} \cdot \vec{\nabla} V_{i} \partial \Omega=-z_{i}\left(\int_{\Omega} c_{0} f V_{i} \partial \Omega+\int_{\Gamma e} \zeta f V_{i} \partial \Gamma\right)
$$

To obtain the same order of magnitude for the two terms $\int_{\Omega} c_{0} f V_{i} \partial \Omega$ and $\int_{\Gamma e} \zeta f V_{i} \partial \Gamma$ in Eq. (10), an appropriate choice of the Steklov coefficient $\zeta$ is given by

$$
\zeta=\frac{\int_{\Gamma e} \partial \Gamma}{\int_{\Omega} c_{0} \partial \Omega}
$$

As previously mentioned, the discrete formulation of Eq. (10) is presented in the Appendix. The most effective method for solving it when the matrix are large is the Lanczos method [18]. This technique calculates a limited number of eigenvalues by projecting the problem on a subspace, then solving it by the QR method [19]. So it is possible to obtain the eigenmodes, subset by subset, in the direction of decreasing eigenvalues (with $z i<0$ ). The current version is the implicit restart technique [20], implemented in ARPACK since 1998 [21]. 
This technique calculates the modes sequentially by blocks, in decreasing order of the eigenvalues associated with them. Since the eigenvalue $z_{i}$ is related directly to the time constant $\tau_{i}$ of the eigenmode by

$$
\tau_{i}=-\frac{1}{z_{i}}
$$

the first modes which are computed correspond to the predominant modes, according to a purely temporal criterion. Even if in theory we can obtain $N_{\text {mesh }}$ eigenmodes, in practice a truncation is performed and the technique computes the $N_{0}$ branch modes corresponding to the first more important eigenmodes (with $z i<0$ ).

The normalization of the original eigenmodes $\widehat{V}_{i}$ is obtained using the relation

$$
\forall i, \quad V_{i}=\frac{\widehat{V}_{i}}{\sqrt{\int_{\Omega} c_{0} V_{i}^{2} d \Omega+\int_{\Gamma} \zeta V_{i}^{2} d \Gamma}}
$$

and leads to the following orthogonality properties:

$$
\forall i, j \quad \int_{\Omega} c_{0} V_{i} V_{j} d \Omega+\int_{\Gamma} \zeta V_{i} V_{j} d \Gamma=\delta_{i j}
$$

and

$$
\forall i, j \quad \int_{\Omega} k \nabla V_{i} \nabla V_{j} d \Omega=-z_{i} \delta_{i j}
$$

\subsection{Projection of the Heat Equation on the Branch Base}

The state equation is obtained by replacing in Eq. (5)

1. The thermal field $T$ by its expression presented in Eq. (6)

2. The test functions $f$ by all the eigenmodes of the reduced basis $\widetilde{V}_{j}(M)$

We can write:

$$
\begin{aligned}
\sum_{i=1}^{N_{0}}\left(\int_{\Omega} c V_{i} V_{j} d \Omega\right) \frac{d x_{i}}{d t}= & -\sum_{i=1}^{N_{0}}\left(\int_{\Omega} k \vec{\nabla} V_{i} \cdot \vec{\nabla} V_{j} d \Omega\right) x_{i} \\
& -\sum_{i=1}^{N_{0}}\left(\int_{\Gamma e} h V_{i} V_{j} d \Gamma\right) x_{i} \\
& +\int_{\Omega} \pi V_{j} \mathrm{\partial} \Omega+\int_{\Gamma e} h T e V_{j} \mathrm{\partial} \Gamma
\end{aligned}
$$

This expression is named the state problem, in which the unknows are the excitation states $x_{j}(t)$ of the eigenmodes. 
When all parameters are fixed $\left(c_{0}, k_{0}, h_{0}\right.$, and $\left.\pi_{0}\right)$, using the orthogonality properties Eqs. (14) and (15), the previous relation becomes

$$
\begin{aligned}
\frac{d x_{j}}{d t}-\sum_{i=1}^{N_{0}}\left(\int_{\Gamma} e \zeta V_{i} V_{j} d \Gamma\right) \frac{d x_{i}}{d t}= & z_{j} x_{j}-\sum_{i=1}^{N_{0}}\left(\int_{\Gamma e} h_{0} V_{i} V_{j} d \Gamma\right) x_{i} \\
& +\int_{\Omega} \pi_{0} V_{j} d \Omega+\int_{\Gamma e} h_{0} T e V_{j} d \Gamma
\end{aligned}
$$

Then, even if the physical problem is linear, all states are coupled to each other. This is the counterpart of the general aspect of the branch basis. However, this equation underlines the particular role of the diagonal terms, which are more important than the others for the great majority of the modes. In practice, as will be seen in the example given, only a few eigenmodes are characterized by significant coupling.

\subsection{Reduction by Amalgam Method}

From the original branch basis, the goal of the modal reduction is to build a reduced basis $\left(\tilde{z}_{i}, \widetilde{V}_{i}\right)$, the dimensions of which are $\widetilde{N}<<N_{0}$, and we can approach correctly the thermal field by the relation

$$
T(M, t) \approx \sum_{j=1}^{\widetilde{N}} \tilde{x}_{j}(t) \widetilde{V}_{j}(M)
$$

The reduction step is performed by the uncoupling amalgam reduction method. The objective of the method is to be independent from fields of reference temperatures, which is the most-used technique in the reduced model domain (as in the POD and MIM). Detailsare explained in [7], and the main steps of this technique are the following.

1. We work in the modal space in which a reference model is chosen. While being close to the real model, this reference model has to be linear and with parameters independent of time [Eq. (17)].

2. Solving this problem is done by neglecting the terms of coupling which exist for only a small number of modes. An analytical expression is then possible and allows us to obtain the reference states at once.

3. The knowledge of these states then permits us to select the most influent modes (called major modes), by a process of minimization of the error between the complete and the reduced model, always in the modal space.

4. The remaining modes (called minor modes) are added to the major modes, and are balanced by a coefficient. The distribution of the minor modes and the choice of the balance coefficients are obtained by the same process of minimization of the error as previously. After normalization [Eq. (13)], we obtain $\widetilde{N}$ reduced modes which possess the same orthogonality properties as the branch modes [Eqs. (14) and (15)]. 
We obtain finally a reduced state problem, similar to Eq. (16), for which the number of unknowns is $\widetilde{N}<<N_{0}$ :

$$
\begin{aligned}
\sum_{i=1}^{\tilde{N}}\left(\int_{\Omega} c \widetilde{V}_{i} \widetilde{V}_{j} \partial \Omega\right) \frac{\partial \tilde{x}_{i}}{\partial t}= & -\sum_{i=1}^{\tilde{N}}\left(\int_{\Omega} k \vec{\nabla} \widetilde{V}_{i} \cdot \vec{\nabla} \widetilde{V}_{j} \partial \Omega\right) \tilde{x}_{i}-\sum_{i=1}^{\tilde{N}}\left(\int_{\Gamma e} h \widetilde{V}_{i} \tilde{V}_{j} \partial \Gamma\right) \tilde{x}_{i} \\
& +\int_{\Omega} \pi \widetilde{V}_{j} \partial \Omega+\int_{\Gamma e} h T e \widetilde{V}_{j} \partial \Gamma
\end{aligned}
$$

\section{THE SUBSTRUCTURING METHOD}

\subsection{The Substructuring Finite Problem}

We consider now the same geometry but dissociated into $N d$ subdomains $\Omega^{(k)}$, as presented in Figure 1:

$$
\Omega=\bigcup_{k=1}^{N d} \Omega^{(k)}
$$

In this case, for the whole domain $\Omega$, there are $N c$ interfaces $\Gamma c^{(l)}, 1 \leq l \leq N c$, which are contiguous to two subdomains $\Omega^{(i)}$ and $\Omega^{(j)}$. By considering for each subdomain $\Omega^{(k)}$ a flux condition on the internal boundary $\Gamma c^{(k)}$, the weak formulation of the heat equation on the subdomain is

$$
\begin{aligned}
\int_{\Omega^{(k)}} c \frac{\partial T^{(k)}}{\partial t} f^{(k)} \partial \Omega= & -\int_{\Omega^{(k)}} k \vec{\nabla} f^{(k)} \cdot \vec{\nabla} T^{(k)} \partial \Omega-\int_{\Gamma e^{(k)}} h T^{(k)} f^{(k)} \partial \Gamma \\
& +\int_{\Omega^{(k)}} \pi f^{(k)} \partial \Omega+\int_{\Gamma e^{(k)}} h T e f^{(k)} \partial \Gamma+\int_{\Gamma c^{(k)}} \varphi^{(k)} f^{(k)} \partial \Gamma
\end{aligned}
$$

with $\varphi^{(k)}=\vec{\varphi}^{(k)} \cdot \vec{n}^{(k)}$ the projection of the flux $\vec{\varphi}^{(k)}$ which is going out of the subdomain $\Omega^{(k)}$ through the internal boundary $\Gamma c^{(k)}$, along the normal $\vec{n}^{(k)}$ to this boundary.

Because through each contiguous boundary $\Gamma c^{(l)}$ there is a flux transfer for both subdomains $\Omega^{(i)}$ and $\Omega^{(j)}$, the weak formulation of the heat equation for the

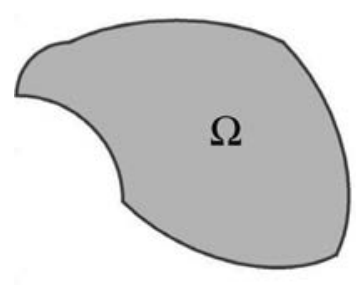

(a)

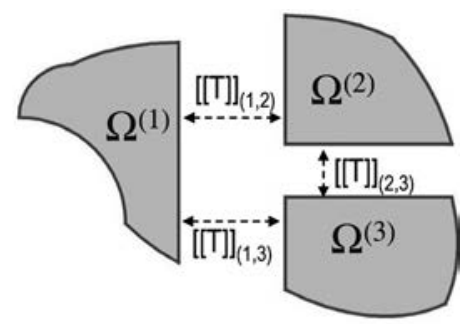

(b)

Figure 1. Substructuring principle: (a) initial geometry; $(b)$ subdomains coupling. 
whole domain $\Omega$ is written as follows:

$$
\begin{aligned}
\sum_{k=1}^{N d} \int_{\Omega^{(k)}} c \frac{\partial T^{(k)}}{\partial t} f^{(k)} \partial \Omega= & -\sum_{k=1}^{N d}\left(\int_{\Omega^{(k)}} k \vec{\nabla} f^{(k)} \cdot \vec{\nabla} T^{(k)} \partial \Omega+\int_{\Gamma e^{(k)}} h T^{(k)} f^{(k)} \partial \Gamma\right) \\
& +\sum_{k=1}^{N d}\left(\int_{\Omega^{(k)}} \pi f \partial \Omega+\int_{\Gamma e^{(k)}} h T e f^{(k)} \partial \Gamma\right) \\
& +\sum_{l=1}^{N c} \int_{\Gamma c^{(l)}}\left(\varphi^{(i)} f^{(i)}+\varphi^{(j)} f^{(j)}\right) \partial \Gamma
\end{aligned}
$$

Given the conservation of heat flux, the latter is connected to the temperatures through the introduction of a thermal contact resistance TCR:

$$
\varphi^{(i)}=-\varphi^{(j)}=\frac{T^{(i)}-T^{(j)}}{T C R} \equiv \frac{[[T]]_{(i, j)}}{T C R}
$$

where the temperature jump $[[T]]_{(i, j)}$ is a surface field, which is defined on all borders contiguous to the different subdomains. This thermal field is unknown.

The weak formulation can finally be written as follows:

$$
\begin{aligned}
\sum_{k=1}^{N d} \int_{\Omega^{(k)}} c \frac{\partial T^{(k)}}{\partial t} f^{(k)} \partial \Omega= & -\sum_{k=1}^{N d}\left(\int_{\Omega^{(k)}} k \vec{\nabla} f^{(k)} \cdot \vec{\nabla} T^{(k)} \partial \Omega+\int_{\Gamma e^{(k)}} h T^{(k)} f^{(k)} \partial \Gamma\right) \\
& +\sum_{k=1}^{N d}\left(\int_{\Omega^{(k)}} \pi f \partial \Omega+\int_{\Gamma e^{(k)}} h T e f^{(k)} \partial \Gamma\right) \\
& +\sum_{l=1}^{N c} \int_{\Gamma c^{(l)}} \frac{[[T]]_{(i, j)}[[f]]_{(i, j)}}{T C R} \partial \Gamma
\end{aligned}
$$

In this way, a temperature jump function appears in the weak formulation.

\subsection{Building of the Substructuring Reduced Basis}

The partition of the domain $\Omega$ into $N d$ subdomains $\Omega^{(k)}$, where each of them is bounded by $\Gamma^{(k)}$, permits us to compute a branch basis $\left(z_{i}^{(k)}, V_{i}^{(k)}\right)$ for each subdomain [Eq. (10)]. Each basis is constituted of $N_{0}^{(k)}$ modes. Unlike other bases, the advantage of using branch modes for the substructuring technique is that the connection between the subdomains is easy, because these modes form a basis for any thermal problem, whatever the boundary conditions are. This substructuring basis allows us then to respect the boundary conditions of temperature jumps imposed by Eq. (24).

The reduction of each basis corresponding to a subdomain is performed by following the uncoupling amalgam method as defined for the classical global BERM method. For each subdomain $\Omega^{(k)}$, we then obtain a reduced basis $\left(\tilde{z}_{i}^{(k)}, \widetilde{V}_{i}^{(k)}\right)$, the dimension of which is $\widetilde{N}^{(k)}$. The extension of each basis on the whole domain 
$\Omega$ is obtained by extending each eigenvector by zero on the other subdomains. Each vector then defined on the whole domain can be written as $\widehat{V}_{i}^{(k)}$. The substructuring reduced basis of the whole domain $\Omega$ is then constituted by all modes $\left(\widehat{z}_{p}, \widehat{V}_{p}\right)$, defined as above. We can then write that all $N d$ thermal fields of each subdomain $\Omega^{(k)}$ can be projected on the substructuring modal basis thus defined as

$$
T^{(k)}(M, t)=\sum_{k=1}^{N d} \sum_{i=1}^{\widetilde{N}^{(k)}} \tilde{x}_{i}^{(k)}(t) \quad \widetilde{V}_{i}^{(k)}(M)=\sum_{p=1}^{\widehat{N}} \widehat{x}_{p}(t) \widehat{V}_{p}(M)
$$

with

$$
\widehat{N}=\sum_{k=1}^{N d} \widetilde{N}^{(k)}
$$

\subsection{The Substructuring State Equation}

The substructuring modal formulation is made by using a method similar to that used for the classical global BERM method [Eqs. (5)-(16)]: The implantation of the temperature projection on the basis [Eq. (25)] in the thermal variational formulation [Eq. (24)], and the use as test function of the eigenvector of the substructuring basis lead to the substructuring state equation.

By considering, for example, the first term (the inertial term) which appears in Eq. (24), it is possible to reassemblate the different subdomains $\Omega^{(k)}$ to obtain the complete domain $\Omega$, because the eigenvectors $\widehat{V}_{p}$ used in the state equation are nonzero only for one subdomain. Thus we write:

$$
\begin{aligned}
\sum_{k=1}^{N d} \int_{\Omega^{(k)}} c \frac{\partial T^{(k)}}{\partial t} f^{(k)} \partial \Omega & =\sum_{p=1}^{\widehat{N}} \sum_{k=1}^{N d}\left(\int_{\Omega^{(k)}} c \widehat{V}_{p} \widehat{V}_{q} \partial \Omega\right) \frac{\partial \widehat{x}_{p}}{\partial t} \\
& =\sum_{p=1}^{N}\left(\int_{\Omega} c \widehat{V}_{p} \widehat{V}_{q} \partial \Omega\right) \frac{\partial \widehat{x}_{p}}{\partial t}
\end{aligned}
$$

The same consideration for the other terms in Eq. (24) leads to the following relation:

$$
\begin{aligned}
\sum_{p=1}^{\widehat{N}}\left(\int_{\Omega} c \widehat{V}_{p} \widehat{V}_{q} \partial \Omega\right) \frac{\partial \widehat{x}_{p}}{\partial t}= & -\sum_{p=1}^{\widehat{N}}\left(\int_{\Omega} k \vec{\nabla} \widehat{V}_{p} \cdot \vec{\nabla} \widehat{V}_{q} \partial \Omega+\int_{\Gamma e} h \widehat{V}_{p} \widehat{V}_{q} \partial \Gamma\right) \widehat{x}_{p} \\
& +\int_{\Omega} \pi \widehat{V}_{q} \partial \Omega+\int_{\Gamma e} h T e \widehat{V}_{q} \partial \Gamma \\
& +\sum_{p=1}^{\widehat{N}}\left(\int_{\Gamma c} \frac{\left[\left[\widehat{V}_{p}\right]\right]_{(i, j)}\left[\left[\widehat{V}_{q}\right]\right]_{(i, j)}}{T C R} \partial \Gamma\right) \widehat{x}_{p}
\end{aligned}
$$


This system is defined by blocks, where each block corresponds to a substructure. The coupling between the different substructure is obtained by the temperature jump term which exists only between two substructures.

\section{APPLICATION}

\subsection{Presentation of the Physical Problem}

The heterogeneous studied system is composed of three subdomains (see Figure 2). The first element $\Omega^{(1)}$ is a parallelepiped block $(9.8 \mathrm{~cm} \times 9.8 \mathrm{~cm} \times 16.4 \mathrm{~cm})$ composed of steel (thermal conductivity $k_{1}=52 \mathrm{~W} / \mathrm{m} \mathrm{K}$ and volume thermal capacity $\left.c_{1}=3,840.10^{3} \mathrm{~J} / \mathrm{m}^{3} \mathrm{~K}\right)$. There is no volume heat source in this subdomains $\left(\pi_{1}=0\right)$.

The other subdomains $\Omega^{(2)}$ and $\Omega^{(3)}$ are two cylindrical heat sources, of diameter $D=20 \mathrm{~mm}$ and length $L=60 \mathrm{~mm}$, which are placed in the block. They are characterized by a thermal conductivity $k_{2}=k_{3}=8 \mathrm{~W} / \mathrm{mK}$ and a thermal capacity $c_{2}=c_{3}=4,200.10^{3} \mathrm{~J} / \mathrm{m}^{3} \mathrm{~K}$. The volume heat sources $\pi_{2}$ and $\pi_{3}$ time evolutions are presented on Figure 3. We suppose that there is no contact resistance between the cylindrical heat sources and the block, defined by the surfaces $\Gamma_{1 i}=\Omega^{(1)} \cap \Omega^{(i)}, i=1,2$.

The two largest vertical faces of the block $\Gamma_{2}$ which are parallel to the $X Z$ plan (see Figure 2) are painted in black and exchange heat with the ambiant (considered at $T_{a}=300 \mathrm{~K}$ ), by convection $\left(h_{a}=8 \mathrm{~W} / \mathrm{m}^{2} \mathrm{~K}\right.$ ), and radiation (emissivity $\varepsilon=0.9$ ).

The block is drilled in its length by two circular ducts (diameter $D=1.6 \mathrm{~cm}$ ). Oil circulates there, with a constant temperature $T_{h}=300 \mathrm{~K}$. The boundary $\Gamma_{3}$ defined between the block and the oil is characterized by a convection coefficient $h_{h}=40 \mathrm{~W} / \mathrm{m}^{2}$.

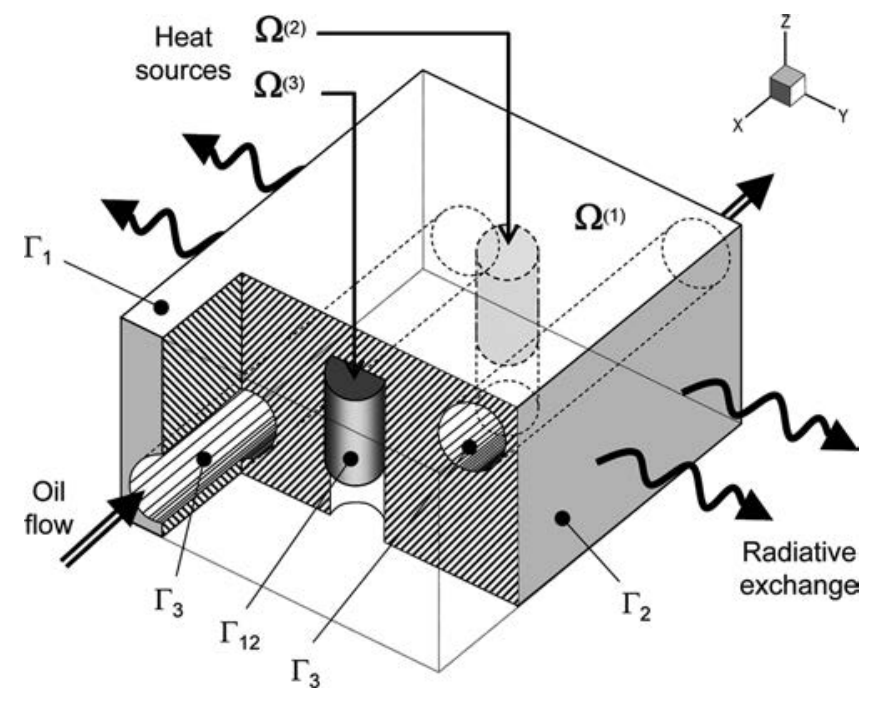

Figure 2. Studied geometry. 


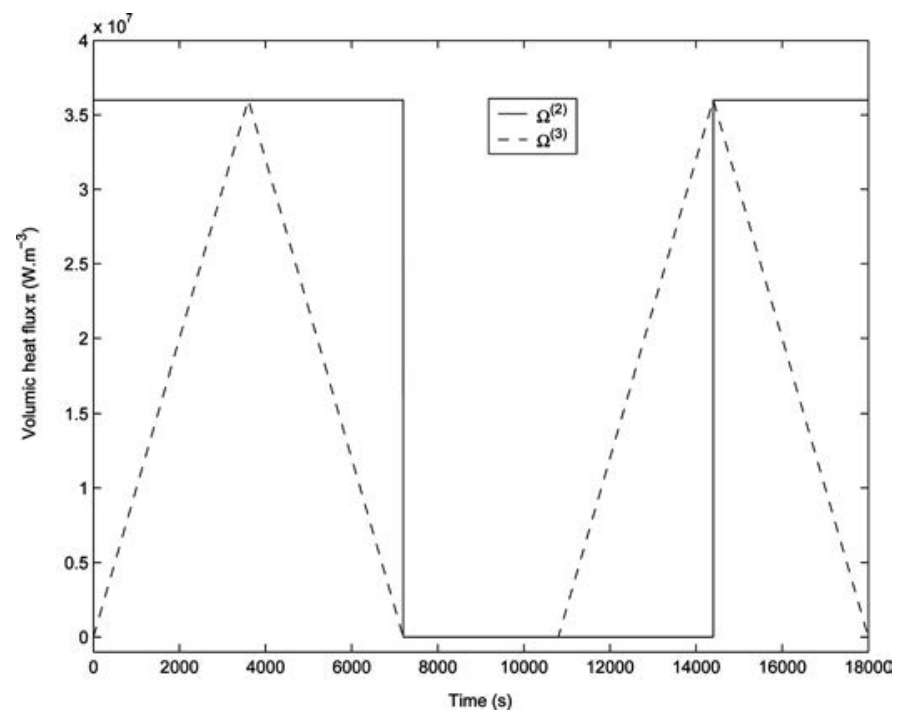

Figure 3. Time evolutions of the volumic heat sources.

All other external surfaces of the block are insulated and are notified $\Gamma_{1}$ (i.e., the faces of $\Omega^{(1)}, \Omega^{(2)}$, and $\Omega^{(3)}$, which are parallel to $X Y$, and the faces of $\Omega^{(1)}$ parallel to $Y Z$ ).

The equations defining the problem are presented below.

$$
\begin{gathered}
\forall M \in \Omega^{(i)}, 1 \leq i \leq 3 \quad c_{i} \frac{\partial T^{(i)}}{\partial t}=\operatorname{div}\left(k_{i} \vec{\nabla} T^{(i)}\right)+\pi_{i} \\
\forall M \in \Gamma_{1} \quad k_{1} \vec{\nabla} T^{(1)} \cdot \vec{n}^{(1)}=0 \\
\forall M \in \Gamma_{2} \quad-k_{1} \vec{\nabla} T^{(1)} \cdot \vec{n}^{(1)}=h_{a}\left(T^{(1)}-T_{a}\right)+\varepsilon \sigma\left[\left(T^{(1)}\right)^{4}-T_{a}^{4}\right] \\
\forall M \in \Gamma_{3} \quad-k_{1} \vec{\nabla} T^{(1)} \cdot \vec{n}^{(1)}=h_{h}\left(T^{(1)}-T_{h}\right) \\
\forall M \in \Gamma_{1 i}, 2 \leq i \leq 3 \quad-k_{1} \vec{\nabla} T^{(1)} \cdot \vec{n}^{(1)}=-k_{i} \vec{\nabla} T^{(i)} \cdot \vec{n}^{(i)} \quad T^{(1)}=T^{(i)}
\end{gathered}
$$

The application treated here is similar to that presented in [8], for which the BERM technique was used for an inverse problem, in order to identify the heat flux dissipated by the cylindrical heat source $\Omega^{(2)}$ in real time. This application has been also used in [22], this time using the MIM technique, always in order to identify the sources of heat $\Omega^{(2)}$ and $\Omega^{(3)}$. 


\subsection{Results with the Complete Model}

The reference model is the classical finite-elements model, which is characterized by $P 1$ triangular discretization leading to a number of $N_{D}=23,503$ nodes. The resolution of this problem by the classical global method [Eq. (5)] is made after spatial discretization in time via an implicit first-order Euler scheme with adaptive time step. The resulting linear system is solved by the preconditionned BICGSTAB algorithm designed for sparse matrices. Computation needs a CPU time equal to $1,105 \mathrm{~s}$, and leads to a thermal field evolution $T_{\text {ref }}$, which is considered as the reference. Figure 4 presents the thermal field à $t=4,000 \mathrm{~s}$. The profile along the segment $A^{\prime}-A$ is shown on Figure 5. Because of the internal heat flux dissipation in $\Omega^{(2)}$ and $\Omega^{(3)}$ and the difference of conductivity, thermal gradients are very different between the steel block and the cylindrical heat sources.

To characterize the importance of the nonlinearity, another simulation is made by considering a linearized radiation term (around $300 \mathrm{~K}$ ), which leads to an equivalent coefficient $h_{a}+h_{\mathrm{rad}}=13.51 \mathrm{~W} / \mathrm{m}^{2} \mathrm{~K}$. Figure 6 represents the time evolution of the temperature, at the point $(x=0.1372 \mathrm{~m}, y=0.0686 \mathrm{~m}, z=0.0307 \mathrm{~m})$, where the difference between the two models is maximum. So we note the impossibility of simplifying the posed problem.

We define a maximum gap by

$$
\Delta T_{\max }=\operatorname{Max}_{\Omega, t}\left|T_{\text {ref }}\right|-\operatorname{Min}_{\Omega, t}\left|T_{\text {ref }}\right|=654 \mathrm{~K}
$$

This gap permits us to define relative mean and maximum errors, according to the relations

$$
\begin{gathered}
\varepsilon_{\text {max }}=\frac{\operatorname{Max}_{\Omega, t}\left|T_{\text {ref }}-T\right|}{\Delta T_{\max }} \\
\varepsilon_{\text {mean }}=\frac{\frac{1}{N_{\text {time }} V} \sum_{i=1}^{N_{\text {time }}} \int_{\Omega}\left|T_{\text {ref }}-T\right| \partial \Omega}{\Delta T_{\max }}
\end{gathered}
$$

For example, between the reference model and that obtained by considering a linearized radiation term, the maximum error is $\varepsilon_{\max }=17.1 \%$, and the mean error is $\varepsilon_{\text {mean }}=5.8 \%$.

\subsection{The Global BERM Method}

4.3.1. Characteristics of the branch basis. Because the number of nodes is not too large, the studied configuration can be resolved by the global BERM method [described by Eq. (16)]. For this computation, Eq. (11) yields the Steklov coefficient $\zeta=120,000$. Given the hardware used, the number of computed branch modes is limited to $N_{0}=3,000$, and the CPU time is $t_{\mathrm{CPU}}=5,900 \mathrm{~s}$.

Visualization modes shows the existence of two very specific spatial evolutions.

1. Some modes are located in the domain and are quasi-null on the boundaries $\Gamma e$, as presented In Figure 7. These are the volume modes. One can notice the special case of the adiabatic boundaries $\Gamma a$ (characterized by the Steklov number $\zeta=0$ ), 


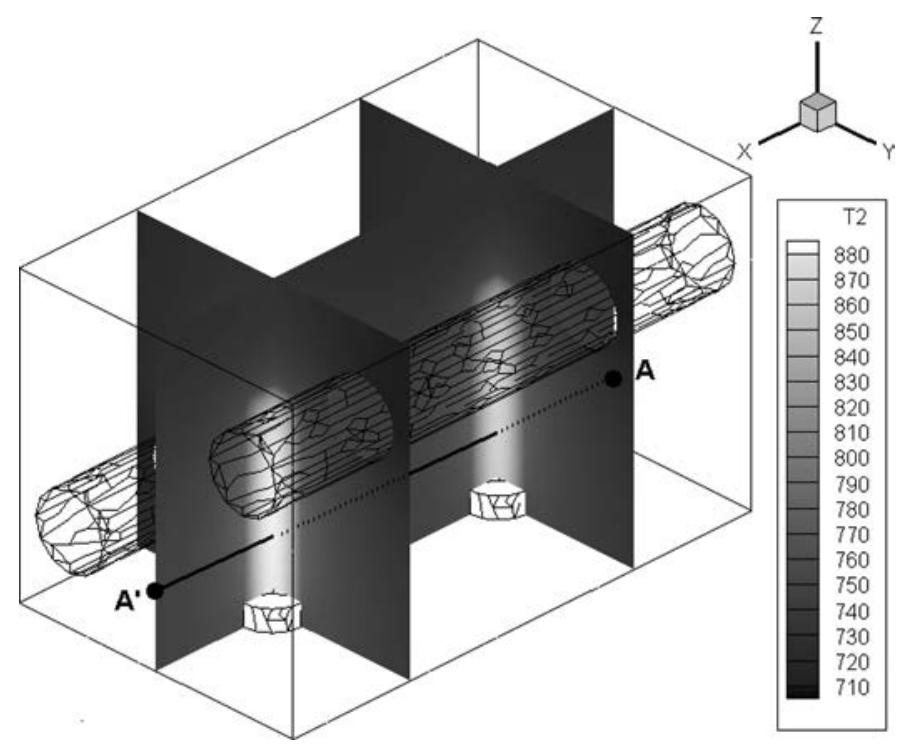

Figure 4. Thermal fied at $t=4,000 \mathrm{~s}$.

appearing as symmetry planes, and for which these volume modes are nonzero. Previous studies have shown that the volume eigenmodes are quasi-periodic fields, the spatial period of which decreases with the eigenvalue, i.e., with the order in which they are computed by the Lanczos method. As we can see in Figure 7 , the isosurfaces pass through the three subdomains indistinguishably.

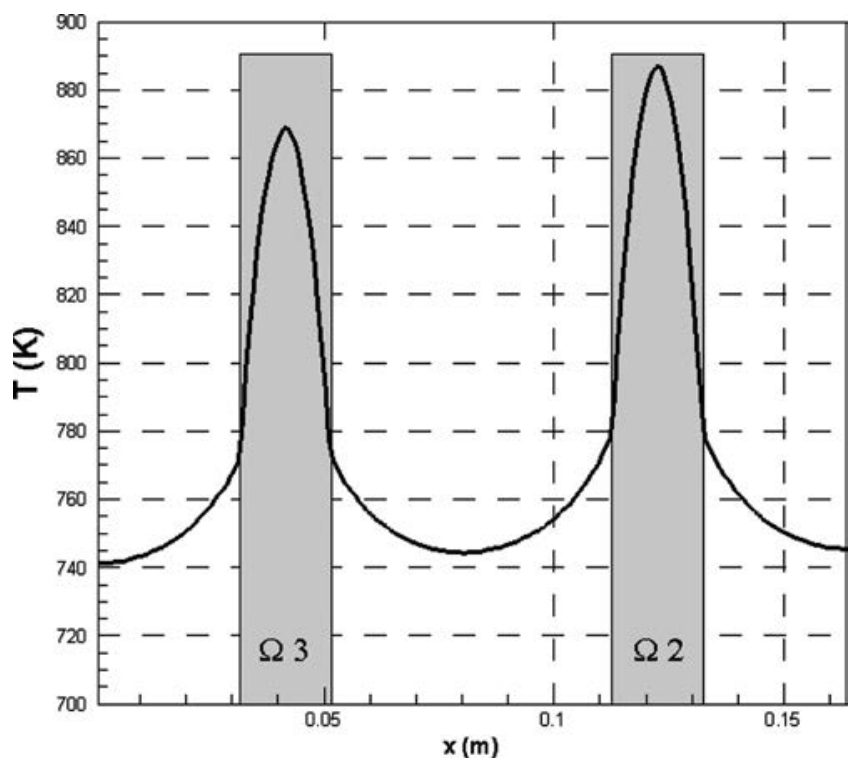

Figure 5. Temperature variations along the segment $\boldsymbol{A}^{\prime} \boldsymbol{A}$ at $t=4,000 \mathrm{~s}$. 


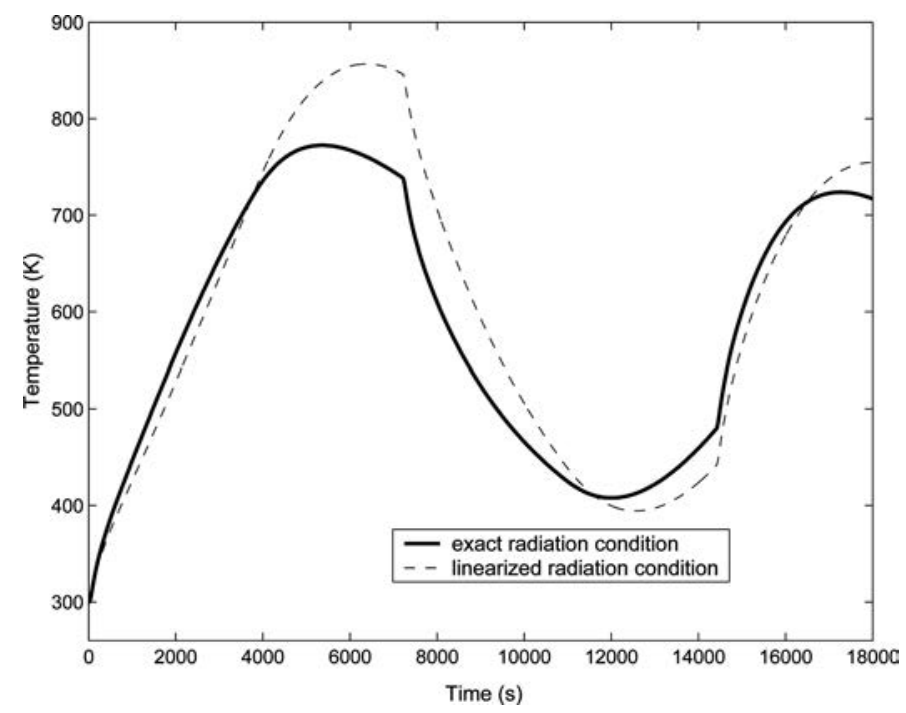

Figure 6. Comparison between the real model and the linearized model.

Thus there is no spatial location by subdomain, even if subdomains are characterized by different thermophysical properties (thermal conductivity here).

2. Other modes are flat on the domain except near the boundaries $\Gamma e$. These are the surface modes. These surface modes originate from the Steklov condition [Eq. (8)]. They are the ones that enable us to consider any type of limit conditions. Figure 8 presents two surface modes, localized at just one boundary. As the volume modes, these surface modes are quasi-periodic fields, and their spatial period decreases with the eigenvalue.

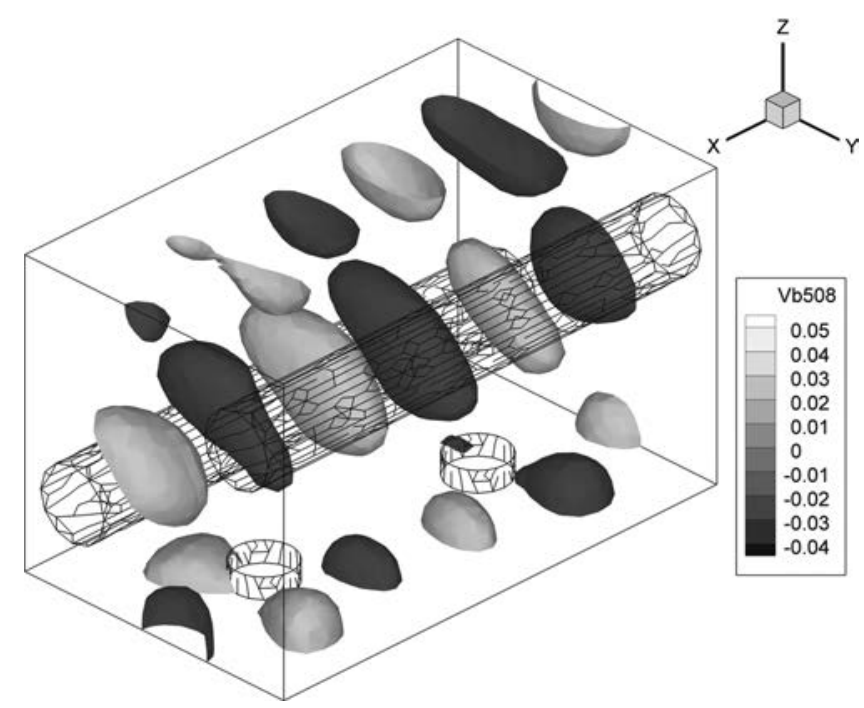

Figure 7. One of the volume modes of the global branch base: representation of two iso-surfaces. 


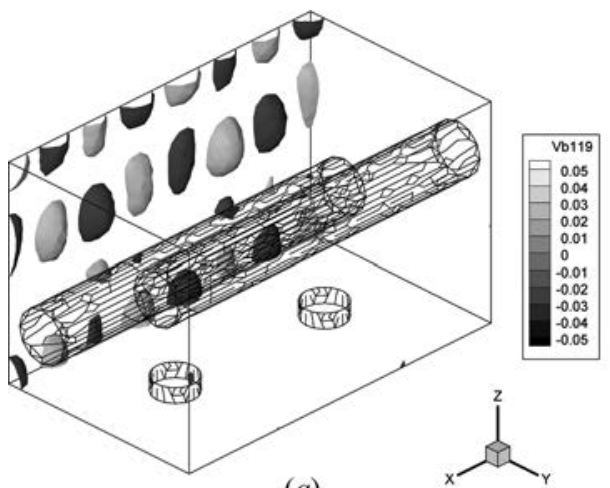

(a)

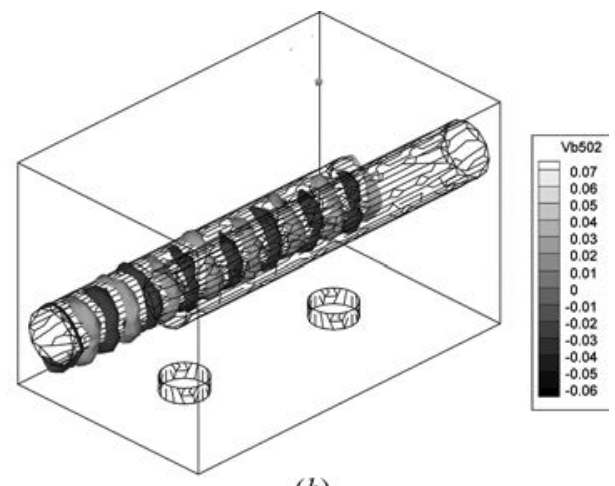

(b)

Figure 8. Surface modes of the global branch base: representation of two iso-surfaces located at (a)boundary $\Gamma_{2}$ and $(b)$ the boundary $\Gamma_{3}$.

An automatic localization of modes can be obtained by the following criterion:

$$
N_{i}^{\text {surf }}=\int_{\Gamma} V_{i} \zeta V_{i} d \Gamma
$$

Because of the orthogonality relation defined in Eq. (14), when $N_{i}^{\text {surf }}$ is close to $0, V_{i}$ is a volume mode, and when $N_{i}^{\text {surf }}$ is close to $1, V_{i}$ is a surface mode. Figure 9 , which presents the $N_{i}^{\text {surf }}$ evolution for the 3,000 branch modes, clearly shows that the great majority of the modes are surface modes. Indeed, given the boundary $\Gamma_{3}$ that runs through the block, the domain modes family cannot be extended easily. Accordingly, many modes are nonzero both on the core of the domain and at the border and are characterized by $0.2<N_{i}^{\text {surf }}<0.8$. These are global modes. This graph also shows

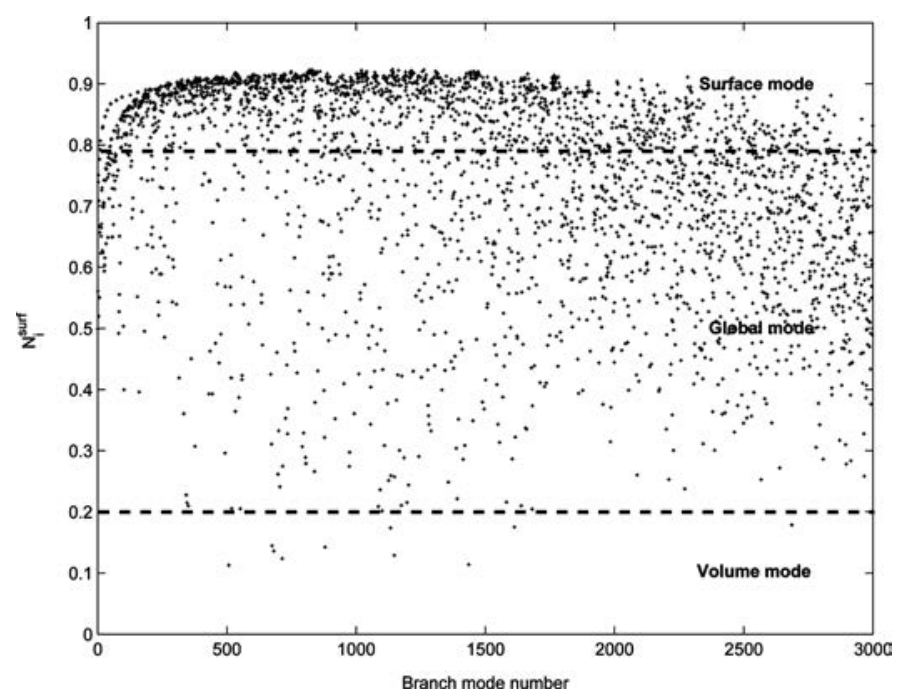

Figure 9. $N_{i}^{\text {surf }}$ criterion evolution for the complete domain $\Omega$. 
clearly that when the branch mode number increases (i.e., $z_{i}<0$ increases), the density of global modes increases at the expense of surface modes, and $N_{i}^{\text {surf }}$ decreases overall.

To reconstitute the important thermal gradient variation observed in the domain near the heat sources (Figure 5), volume and global modes are important. It is then necessary to compute an important number of branch modes, which is not easy when the mesh is large. To determine the error linked to the truncation of the initial Branch basis from which the reduced basis is built, the following process is performed.

1. The projection of the reference thermal field $T^{\mathrm{ref}}(M, t)$ on each vector of the basis $V_{i}(M)$ permits us to obtain the associated states $x_{j}^{\text {ref }}(t)$ :

$$
x_{j}^{\mathrm{ref}}(t)=\int_{\Omega} T^{\mathrm{ref}}(M, t) c V_{j}(M) d \Omega+\int_{\Gamma} T^{\mathrm{ref}}(M, t) \zeta V_{j}(M) d \Gamma
$$

2. These state vectors are used to rebuild the thermal field $T^{r}(M, t)$ :

$$
T^{r}(M, t)=\sum_{j=1}^{N_{0}} x_{j}^{\mathrm{ref}}(t) V_{j}(M)
$$

3. It is then possible to obtain the errors between the thermal field rebuilt and the reference, by using Eqs. (35) and (36).

With 3,000 branch modes, the maximum error computed is equal to $5 K$, i.e., $\varepsilon_{\max }=0.6 \%$. So we consider that the truncated base of 3,000 modes is sufficient to reconstruct the temperature fields satisfactorily. It is from this basis that the reduced bases are then built.

4.3.2. Results of the global BERM method. Some reduced models are then built from the branch modes by considering the following reference model:

1. For each cylindrical heat source, we consider a volume power $\pi_{\max }=$ $3.6 \times 10^{7} \mathrm{~W} / \mathrm{m}^{3}$.

2. For the steel block, there is no volume flux dissipation. External boundary conditions correspond to the physical problem with linearization of the radiation term (when it exists). We use then for $\Gamma_{2}, h=30 \mathrm{~W} / \mathrm{m}^{2}$ and $T_{\text {ext }}=300 \mathrm{~K}$ and for $\Gamma_{3}, h=40 \mathrm{~W} / \mathrm{m}^{2}$ and $T_{\text {ext }}=300 \mathrm{~K}$.

Different reduced models are then built, with $50<\widetilde{N}<300$. Because of the analytical technique, the CPU time is negligible in comparison with the CPU time for the initial branch basis computation.

From these reduced bases, the different state equations systems [Eq. (19)] are then built and evolved in time like the complete model via an implicit first-order Euler scheme with adaptive time steps, but this time the linear systems are solved by a LU algorithm. The results are presented in Table 1 . The global BERM method gives satisfactory overall results: For a reduction order $\widetilde{N}=100$, the average error is 
Table1. Global reduced model: results

\begin{tabular}{lccc}
\hline Global order $\widehat{N}$ & $\varepsilon_{\max }(\%)$ & $\varepsilon_{\text {mean }}(\%)$ & $t_{\text {CPU }}(\mathrm{s})$ \\
\hline 50 & 14.12 & 1.57 & 13 \\
100 & 10.93 & 0.88 & 44 \\
150 & 9.23 & 0.80 & 115 \\
200 & 7.01 & 0.78 & 212 \\
250 & 5.73 & 0.75 & 332 \\
300 & 4.88 & 0.71 & 493 \\
\hline
\end{tabular}

less than $1 \%$, while the gain in computational time is 25 . However, with this model, errors can be locally important $\left(\varepsilon_{\max } \approx 11 \%\right)$. When increasing the model order, the time saved decreases quickly, while the accuracy of the model increases relatively weakly: A reduced model the order of which is $\widetilde{N}=300$ leads to a maximal error $\varepsilon_{\max } \approx 5 \%$, while the gain in CPU time is just over 2.2. As the work of Videcoq et al. [8] showed, this technique leads to precise results for a heat flux identification process, in which complete knowledge of the temperature field is not desired. However, the global BERM method shows these limits when we want accurate results for the temperatures in the entire domain. The reason lies in the low number of volume modes that appear in the calculated basis (which is necessarily truncated because of the hardware limitations). Indeed, as we have seen that these volume modes were not specific for each subdomain, if we have to built a spatial thermal evolution which is very different depending on the considered subdomain (for example, due to the presence of a point source), it is necessary to keep a large number of these modes. Thus the low number of volume modes which is present in the original basis prevents a precise rebuilding of the temperature field in the zones where there are strong variations of temperature gradient, i.e., between two subdomains. The best would be to use modes which are localized by subdomains. That is the principle of the substructuring technique, in which modes are computed by subdomains.

\subsection{The Substructuring Model}

The substructuring BERM method is used, by choosing three subdomains, $\Omega^{(1)}, \Omega^{(2)}$, and $\Omega^{(3)}$.

4.4.1. Substructuring branch basis computation. The computation of the branch basis linked to each subdomain is performed as in Eq. (10). For the subdomains $\left\{\Omega^{(1)}, \Omega^{(2)}, \Omega^{(3)}\right\}$, the Steklov parameter $\zeta$ is fixed respectively to $\{110,000,18,210,18,210\}$, by following the criterion defined in Eq. (11). As previously, the substructuring base for the subdomain $\Omega^{(1)}$ is limited to $N_{0}^{(1)}=3,000$. The computation time is $t_{\mathrm{CPU}}=5,265 \mathrm{~s}$. Concerning the other subdomains $\Omega^{(2)}$ and $\Omega^{(3)}$ corresponding to the two cylindrical heat sources, the computation of the branch basis is easy because of the weak dimension of the meshes, and almost all the modes are calculated $\left(N_{0}^{(2)}=N_{0}^{(3)}=1,470\right)$. For each of them, computation time is $t_{\mathrm{CPU}}=115 \mathrm{~s}$.

This time, surfaces $\Gamma_{12}$ and $\Gamma_{13}$ between the steel block and the heat sources are considered for each subdomain are boundaries, on which surface modes appear 


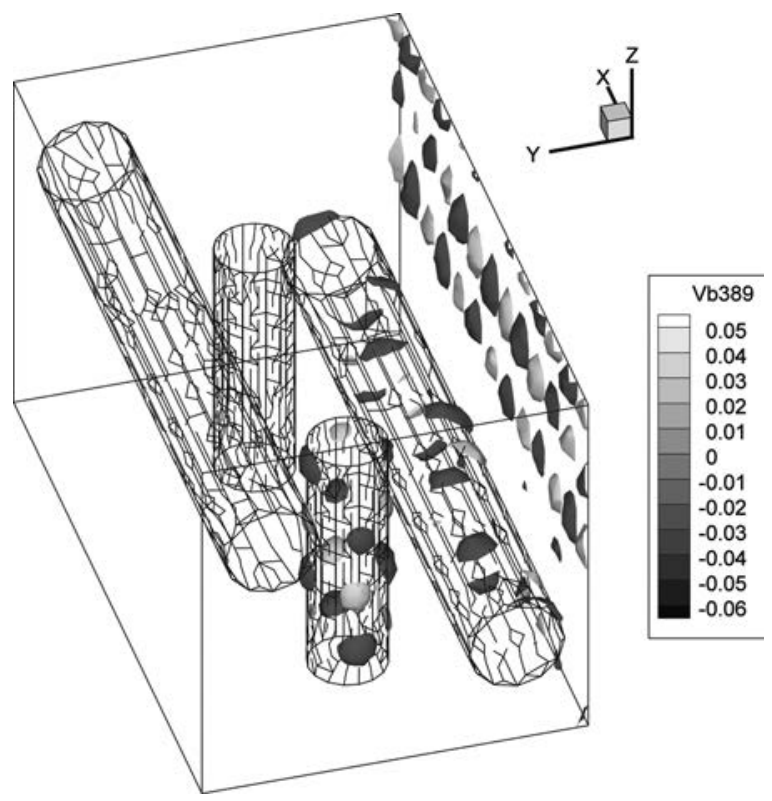

Figure 10. One of the surface modes of the substructuring branch base for the subdomain $\Omega^{(1)}$, representation of two iso-surfaces.

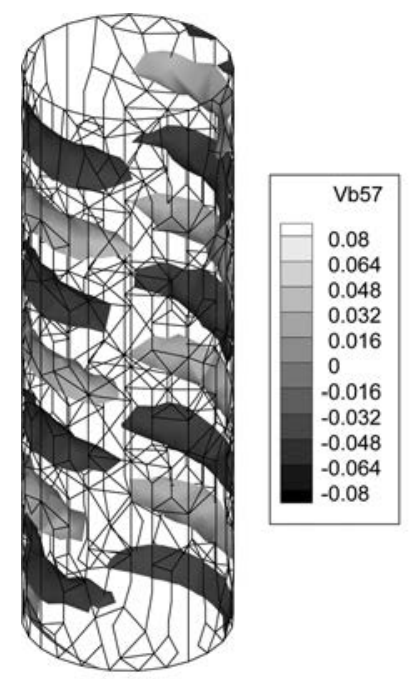

(a)

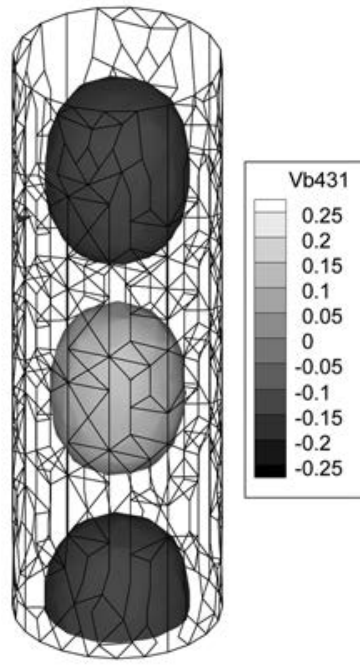

(b)

Figure 11. Modes of the substructuring branch base for the subdomain $\Omega^{(2)}$, representation of two iso-surfaces; $(a)$ surface mode and $(b)$ volume mode. 


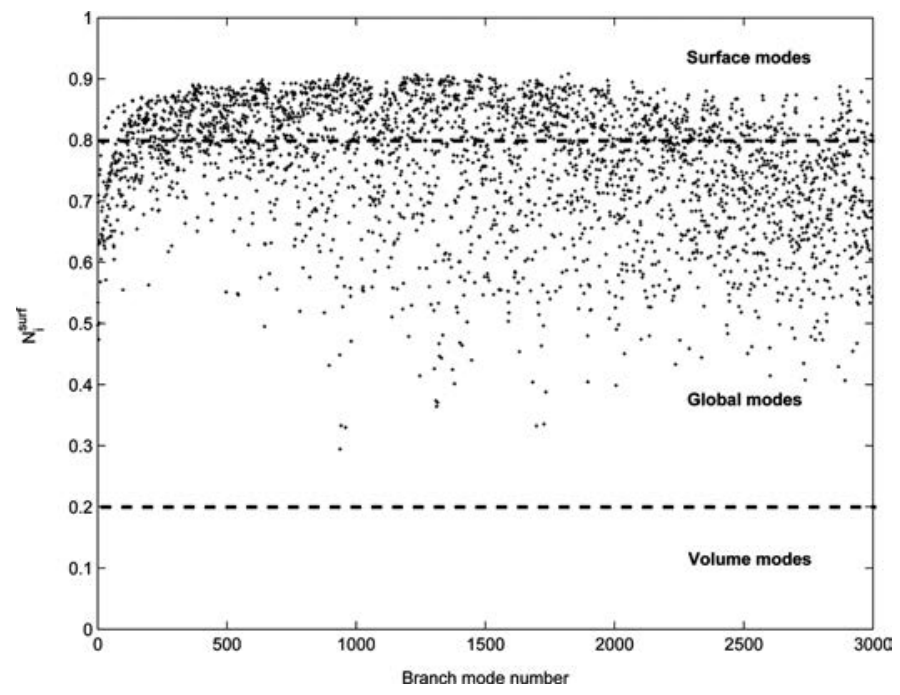

Figure 12. $N_{i}^{\text {surf }}$ criterion evolution for subdomain $\Omega^{(1)}$.

(Figure 10 for the block steel $\Omega^{(1)}$ and Figure 11 a for one cylindrical heat source $\left.\Omega^{(2)}\right)$. Figure 12 presents the $N_{i}^{\text {surf }}$ evolution for the subdomain $\Omega^{(1)}$. The evolution is similar to that obtained in the case of the global basis defined on the whole field $\Omega$ (Figure 9), but this time the number of modes which tend to volume modes is much less important: In the case of the global branch basis, the proportion of modes characterized by $N_{i}^{\text {surf }}<0.4$ is $4.3 \%$, and that corresponding to $N_{i}^{\text {surf }}<0.2$ is $0.3 \%$. In the case of the branch base defined only for the steel block $\Omega^{(1)}$, the proportion of modes characterized by $N_{i}^{\text {surf }}<0.4$ is only $0.3 \%$, and that corresponding to $N_{i}^{\text {surf }}<0.2$ is zero. This is due to boundaries $\Gamma_{1,2}$ and $\Gamma_{1,3}$ crossing the block, which prevents the development of this mode family.

The $N_{i}^{\text {surf }}$ evolution for subdomains $\Omega^{(2)}$ and $\Omega^{(3)}$ is presented in Figure 13. This time, surface modes (Figure 11a) and volume modes (Figure 11b) clearly appear, with just some global modes. A phenomenon even more marked than for the previous evolution of $N_{i}^{\text {surf }}$ is that the first computed modes (corresponding to the larger $\left.z_{i}<0\right)$ are all surface modes.

As presented for the global branch basis [Eqs. (38)-(39)], the precision of each temporary basis constituted by the first eigenvectors $V_{j}^{(k)}$ is computed for all subdomains $1 \leq k \leq 3$, and compared to the precision of the temporary global basis. Results are presented in Figure 14. For all subdomains, results are precise and better than those corresponding to the complete domain $\Omega$.

Indeed, as we have seen that the substruturing technique allows us to obtain surface modes on the external and the internal boundaries, and volume modes limited to each subdomain, the complete substructured basis is far richer than the global basis, and moreover, this richness exists as soon as the first modes of the basis have been computed.

In our application we saw that it was necessary to reconstitute an important variation of thermal gradient near the internal boundaries $\Gamma_{12}$ and $\Gamma_{13}$. For the 


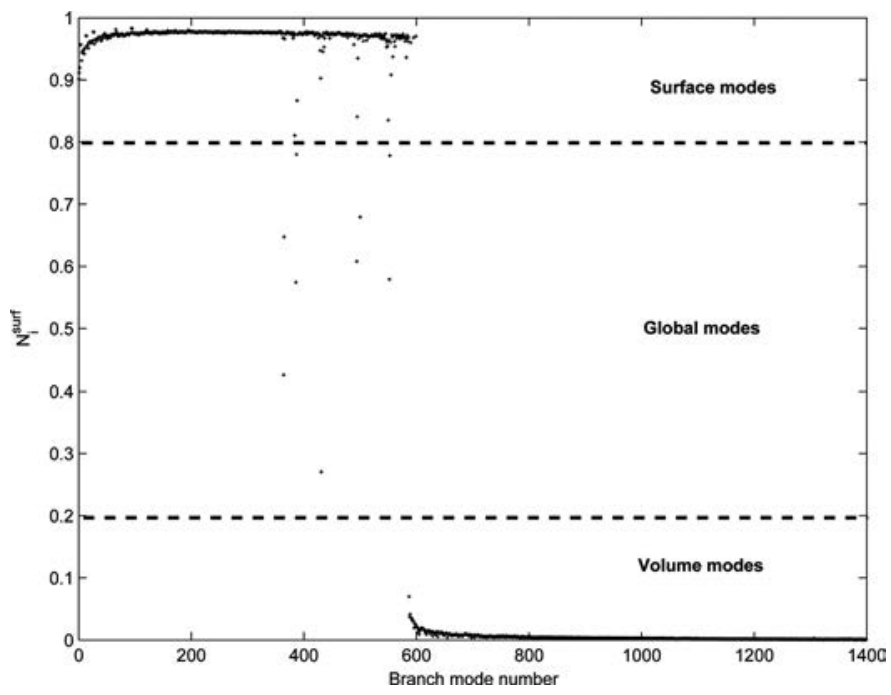

Figure 13. $N_{i}^{\text {surf }}$ criterion evolution for subdomain $\Omega^{(2)}$.

complete domain $\Omega$, these important variations of gradients are located inside the domain, and have to be reconstituted by volume modes which are not numerous. For each subdomain, these gradients can be reconstituted by the surface modes, which are numerous.

To build the final reduced basis, the uncoupling amalgam method is used. The difficulty lies in the choice of the parameters of the Fourier condition at the boundaries $\Gamma_{12}$ and $\Gamma_{13}$. The test case is then completely different from the real problem.

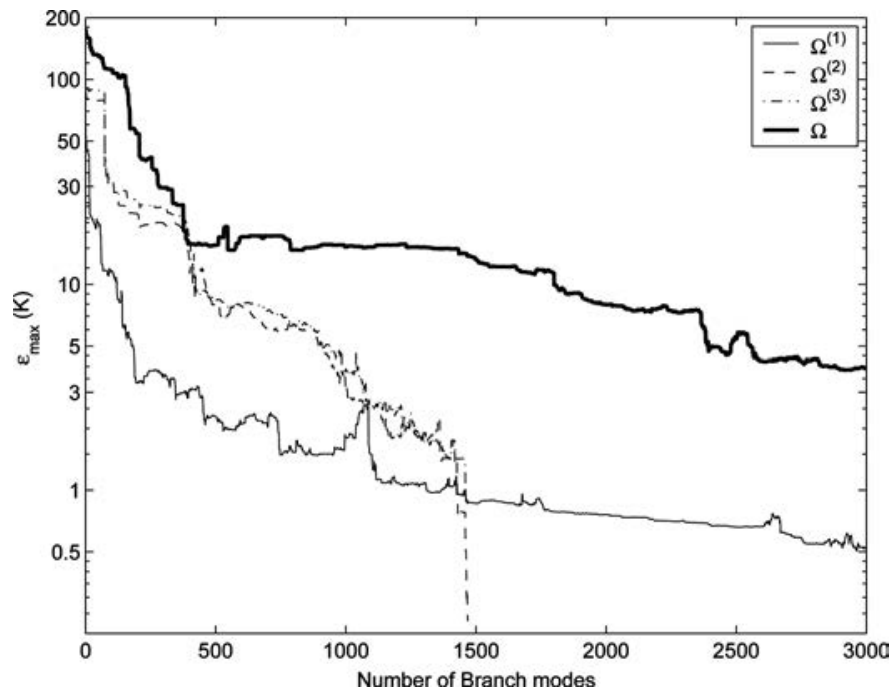

Figure 14. Choice of the minimum number of branch modes for each subdomain for the substructuring configuration $\left(\Omega^{(1)}, \Omega^{(2)}, \Omega^{(3)}\right)$ and for the one-body configuration $(\Omega)$. 
The difficulty is then to choose an optimal linear test case for each subdomain considered as independent of the other subdomains. In [17] it was shown that the precision of the substructuring reduced model did not vary a lot according to the test case used. Indeed, it is not necessary that the temperature fields corresponding to the reference test case be identical to those of the physical problem considered. Even if the these two problems are different, if the diffusion of heat observed in the test case follows approximately the same direction as the diffusion that is obtained in the real case, then the the patterns of temperature fields will be similar. The modes preferentially excited by the test case will then have a dominant role in the reconstruction of temperature fields of the real problem. The reduced model, optimal for the test case, will then be quite satisfactory for the real physical problem.

The different test cases corresponding to each subdomains are presented below.

1. For each cylindrical heat source, we consider as before a volume power $\pi_{\max }=3.6 \times 10^{7} \mathrm{~W} / \mathrm{m}^{3}$. Furthermore, we choose for the exchange coefficient $h=50 \mathrm{~W} / \mathrm{m}^{2}$ and for the external temperature $T_{\text {ext }}=300 \mathrm{~K}$.

2. For the steel block, there is no volume flux dissipation. External boundary conditions are chosen as previously and we then use for $\Gamma_{1}$ and $\Gamma_{2}, h=30 \mathrm{~W} / \mathrm{m}^{2}$ and $T_{\text {ext }}=300 \mathrm{~K}$, and for $\Gamma_{3}, h=40 \mathrm{~W} / \mathrm{m}^{2}$ and $T_{\text {ext }}=300 \mathrm{~K}$. Concerning the contiguous boundaries with the cylindrical heat sources, because of the important volume power they dissipate, we choose an important exchange coefficient $h$. To take into account the difference of the dissipated energy in the two cylindrical heat sources (see Figure 3), the conditions are different for the two boundaries: For $\Gamma_{12}$ we choose $h=10,000 \mathrm{~W} / \mathrm{m}^{2}$, and for $\Gamma_{13}$ we have $h=1,000 \mathrm{~W} / \mathrm{m}^{2}$, with $T_{\text {ext }}=500 \mathrm{~K}$ for the two boundaries.

Different reduced models are then built, with $50<\widehat{\mathrm{N}}<300$. The manner of repartition of the number of eigenmodes corresponding to each subdomain is difficult. At present no precise rule is defined. A first estimation consists of linking it to the minimal number of branch modes to use for each subdomain. As seen previously, we found for the treated application $\left\{N_{0}^{(1)}, N_{0}^{(2)}, N_{0}^{(3)}\right\}=\{3,000,1,470,1,470\}$, which leads to a ratio of 2 between the number of modes of the block and of each cylindrical heat source. For the different models tested, the reduced modes ratio varies from 1 to 3 between the steel block and each cylindrical heat source.

4.4.2. Choice of the fictitious resistance. As shown by Eq. (24), the substructuring method needs to introduce a thermal contact resistance TCR, which does not exist in the real physical configuration. The problem is then to verify whether the introduction of such a fictitious resistance leads to satisfactory results.

To be able to observe the fictitious thermal contact resistance influence independently of the errors coming from the modal reduction, a first step consists of solving the problem in the thermal space. The discretization is performed in such a way that the meshes of the different subdomains correspond to each other on the contiguous internal boundaries. The mesh sizes for the different subdomains $\left\{\Omega^{(1)}\right.$, $\left.\Omega^{(2)}, \Omega^{(3)}\right\}$ are, respectively, $N_{\mathrm{ST}}=\{22,377,1,476,1,479\}$. To choose the thermal contact resistance value, a thermal balance is performed in a simple case: We consider thus the stationnary case in which the maximum thermal power coming from a 
cylindrical heat source $\pi_{\max }=3.6 \times 10^{7} \mathrm{~W} / \mathrm{m}^{3}$ passes through the internal boundary in a uniform way:

$$
\pi_{\max }=\frac{4 \varphi}{D}=\frac{4 \Delta T}{D T C R}
$$

For a fixed relative temperature jump $\epsilon=\Delta T / \Delta T_{\max }=0.25 \%$, the fictitious thermal contact resistance value obtained by Eq. (40) is TCR $=10^{-5} \mathrm{~m}^{2} \mathrm{~K} / \mathrm{W}$. This value corresponds approximately to the value of thermal contact resistance between two reamed surfaces subjected to a pressure of $1 \mathrm{GPa}$.

The resolution of the substructuring finite-element method is then performed as defined by Eq. (24). Results are satisfactory, since the maximum error defined by Eq. (35) is equal to $\varepsilon_{\max }=0.51 \%$. Figure 15 presents the evolution of temperature for the point which corresponds to the maximal error. This point is located in the second cylindrical heat source $\left(\Omega^{(3)}\right)$.

4.4.3. Results. From this TCR value, different reduced models $(50<\widetilde{N}<300)$ are used for the resolution of the real problem. The results are presented in Table 2 and show the interest of the substructuring method: For acceptable precision (i.e., $\varepsilon_{\max }<10 \%$ ), the CPU time is very much smaller than that obtained by the classical model $\left(t_{\mathrm{CPU}}=1,105 \mathrm{~s}\right)$. For a reduced model the order of which is $\widehat{N}=100=\{40,30,30\}$, the CPU gain reaches 23.5, while the maximum error $\varepsilon_{\max } \approx 3 \%$. Figure 16 shows the maximum error evolution, which is located in $\Omega^{(3)}$.

It is important to compare this error with that resulting from the introduction of the fictitious thermal contact resistance $\left(\varepsilon_{\max }=0.51 \%\right)$ : For a reduction order small enough to permit a significant gain of CPU time, the error between the reduced

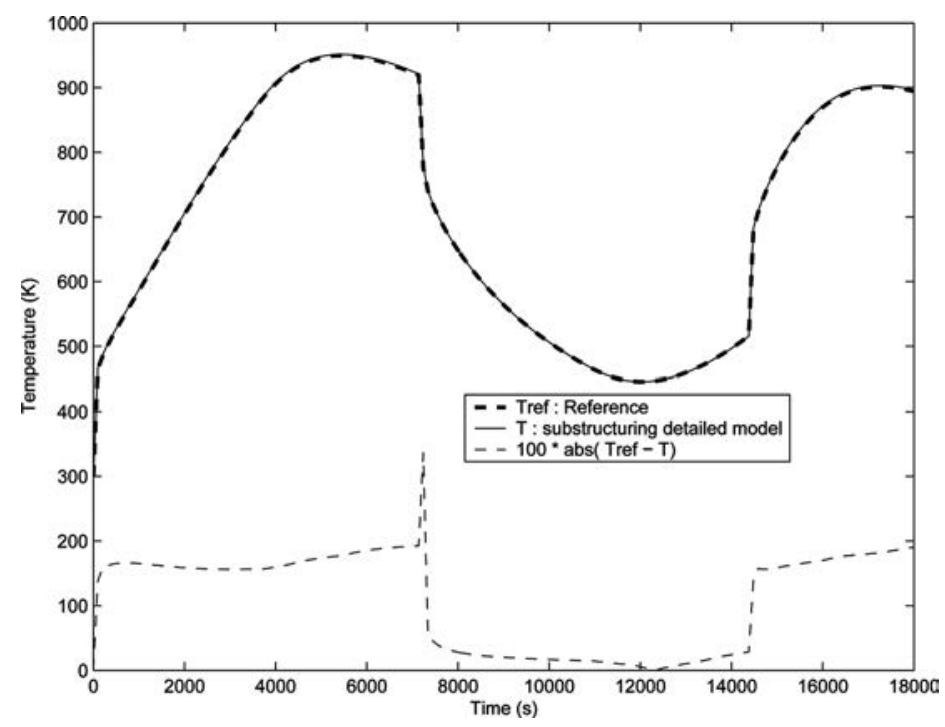

Figure 15. Substructuring detailed model: maximum error evolution. 
Table 2. Susbstructuring reduced model: results

\begin{tabular}{lcccc}
\hline Global order $\widehat{N}$ & $\left\{\tilde{N}^{(1)}, \tilde{N}^{(2)}, \tilde{N}^{(3)}\right\}$ & $\varepsilon_{\max }(\%)$ & $\varepsilon_{\text {mean }}(\%)$ & $t_{\text {CPU }}(\mathrm{s})$ \\
\hline 50 & $\{30,10,10\}$ & 7.46 & 0.72 & 43 \\
100 & $\{40,30,30\}$ & 3.33 & 0.69 & 46 \\
150 & $\{50,50,50\}$ & 3.13 & 0.68 & 58 \\
200 & $\{80,60,60\}$ & 2.99 & 0.67 & 80 \\
250 & $\{90,80,80\}$ & 2.85 & 0.67 & 104 \\
300 & $\{100,100,100\}$ & 2.80 & 0.66 & 138 \\
\hline
\end{tabular}

substructuring model and the reference finite-element model is especially due to the reduction and not to the fictitious thermal contact resistance.

The improvement provided by the substructuring BERM method, as compared to the global BERM method, is indisputable when comparing Tables 1 and 2.

First, when the orders of the reduced models are the same, and when these are high enough, the computation times for the substructuring BERM method are significantly lower than those obtained by the global BERM method. Indeed, since each eigenvector is initially calculated for only one subdomain and extended by zero on the other subdomains, the equation of state (28) is characterized by sparse matrix per block, making its resolution faster.

Moreover, and most important, when the reduction order is the same, the precision obtained by solving the substructuring modal formulation is better than that produced by the global method. This is due to all that has been observed previously, i.e., the substructuring branch basis is more appropriate compared to the global branch basis, when reconstructing temperature fields for subdomains characterized by very different properties and/or different thermal solicitations, which generate large variationsin temperature gradients at the contiguous borders.

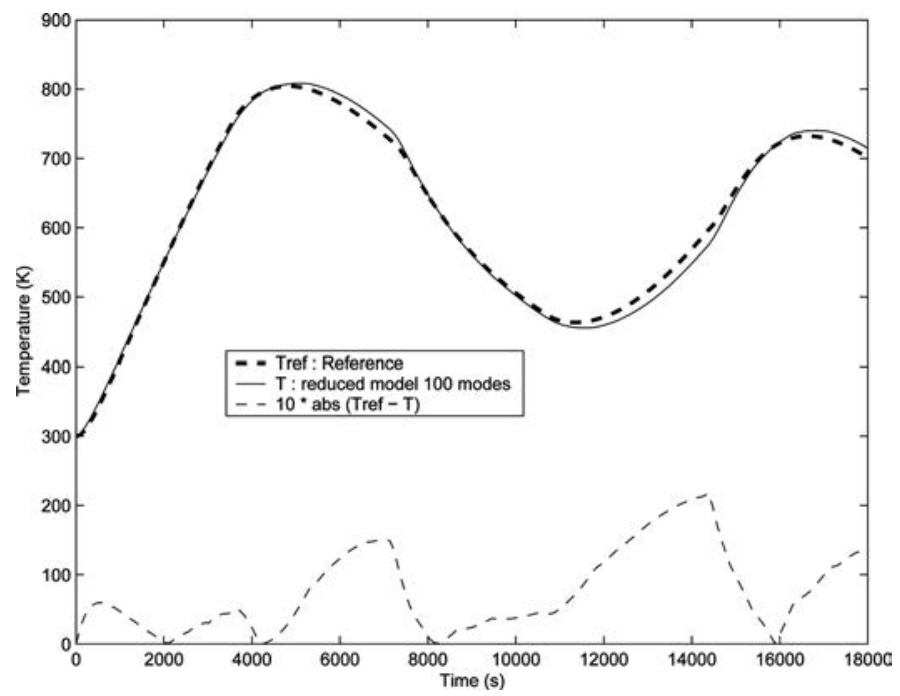

Figure 16. Substructuring reduced model: maximum error evolution. 
Finally, a substructuring modal model the order of which is 100 leads to precision equal to $3 \%$ and a CPU time gain of 23.5, while the global BERM model the order of which is 300 leads to a precision equal to $5 \%$, for a CPU time gain just equal to 2. Then, even if it is possible to use a global BERM model, a substructuring technique is more efficient in the case studied.

\section{CONCLUSION}

The use of the substructuring technique for the resolution of the reduced modal model of the nonlinear 3-D thermal problem posed in this study permitted us to show the following points.

First, the branch basis is especially adapted to the substructuring technique, because a large number of surface modes appear naturally at the boundaries of each subdomains, which allows easy coupling between them.

The technique of reduction of the branch base by the uncoupled amalgam technique is well adapted to the substructured formulation, even if the reference problem of each subdomain is necessarily different from the real global problem.

Given its principle, the substructuring method naturally treats the problems of imperfect contacts between subdomains. We have seen here that this method can also solve problems for which the contacts are either perfect or the partition is fictitious. Indeed, the fictitious thermal contact resistance introduced in the substructuring model does not lead to important bias, in comparison with that resulting from the reduction step. The TCR value can be easily obtained from physical considerations, and the numerical value obtained corresponds physically to the conditions of excellent contact. It is then possible to create fictitious internal boundaries to define subdomains, the mesh size of each being small enough that a branch basis can be computed.

In the case where a global BERM method is possible, the use of a substructuring model can still be attractive, in particular when the different subdomains are characterized by different thermal behaviors.

So the substructuring technique allows us to extend the BERM method. This modal method has proven its efficiency for homogeneous domains characterized by complex geometries, but cases of high variations of the thermal properties are not easy to solve by modal formulation. It is now possible, by using this method, to resolve a lot of thermal problems, which can be nonlinear and with unstationary parameters, characterized by complex geometries leading to important meshes sizes. The modal method then becomes more adapted to real problems corresponding to industrial cases. The next step is to develop this technique in configurations where the domains are in relative motion, for which the modal formulation of branches has already demonstrated interest [9].

\section{REFERENCES}

1. J. Saigon and A. Neveu, Application of Modal Analysis to Modelling of Thermal Bridge in Building, Energy and Buildings, vol. 10, pp. 109-120, 1987.

2. O. Quéménér, J.-L. Battaglia, and A. Neveu, Resolution d'un Probleme Inverse Par Utilisation d un Modélé Réduit Modal, Application au Frottement d un Pion sur un Disque en Rotation, Int. J. Thermal Sci, vol. 42, pp. 361-378, 2003. 
3. M. Girault and D. Petit, Identification Method in Non-linear Heat Conduction. Part 1: Modal Reduction, Int. J. Heat Mass Transfer, vol. 48, pp. 105-118, 2005.

4. O. Balima, Y. Favennec, and D. Petit, Model Reduction for Heat Conduction with Radiative Boundary Conditions Using the Modal Identification Method, Numer. Heat. Transfert B, vol. 52, pp. 107-130, 2007.

5. D. Blinov, V. Prokopov, Y. Sherenkovskii, N. Fialko, and V. Yurchuk, Effective Method for Construction of Low-Dimensional Models for Heat Transfer Process, Int. J. Heat Mass Transfer, vol. 47, pp. 5823-5828, 2004.

6. A. Fic and R. Bialecki, Solving Transient Non Linear Heat Conduction Problem by Proper Orthogonal Decomposition and FEM, Proceeding of CHT-04-173, pp. 67, 2004.

7. O. Quéménér, A. Neveu, and E. Videcoq, A Specific Reduction Method for Branch Modal For-mulation: Application to Highly Non Linear Configuration, Int. J. Thermal Sci., vol. 46, pp. 890-907, 2006.

8. E. Videcoq, O. Quéménér, M. Lazard, and A. Neveu, Heat Source Identification and On-line Temperature Control by a Branch Eigenmodes Reduced Model, Int. J. Heat Mass Transfer, vol. 51, pp. 4743-4752, 2008.

9. F. Joly, O. Quéménér, and A. Neveu, Modal Reduction of an Advection-Diffusion Model Using a Branch Basis," Numer. Heat. Transfert B, vol. 53, pp. 466-485, 2008.

10. L. Baeza, A. Roda, and J. Nielsen, Railway Vehicle/Track Interaction Analysis Using a Modal Substructuring Approach, J. Sound Vibration, vol. 293, pp. 112-124, 2006.

11. V. Faucher and A. Combescure, A Time and Space Mortar Method for Coupling Linear Modal Subdomains and Non-Linear Subdomains in Explicit Structural Dynamics, Comput. Meth. Appl. Mech. Eng., vol. 192, pp. 509-533, 2003.

12. A. Pantano and R. C. Averillc, A Penalty-Based Interface Technology for Coupling Independently Modeled 3d Finite Element Meshes, Finite Elem. Anal. Design, vol. 43, pp. 271-286, 2007.

13. G. Xiao, L. Zhang, and Y. Zhou, Generalized Heat Transition Matrix for Arbitrarily Shaped Thermal Media and Its Applications to Steady-State Heat Conduction Problems in Large-Scale Systems, Numer. Heat Transfer, Part B, vol. 59, pp. 319-338, 2011.

14. D. Petit and R. Hachette, Model Reduction in Linear Heat Conduction: Use of Interface Fluxes for the Numerical Coupling, Int. J. Heat Mass Transfer, vol. 41, pp. 3177-3189, 1998.

15. A. El-Biyaali, J. Roux, and C. Inard, Sous Structuration Et Couplage De Modele Reduits Appliques Aux Transferts Thermiques Dans Le Sol, Int. Commun. Heat Mass Transfer, vol. 23 , pp. 575-586, 1996.

16. B. Flaments, F. Bourquin, and A. Neveu, Synthese Modale, Une Méthode de Sous Structuration Dynamique Pour La Modelisation des Systemes Thermiques Linéaires, Int. J. Heat Mass Transfer, vol. 36, pp. 1649-1662, 1993.

17. P. Laffay, O. Quéménér, and A. Neveu, Developing a Method for Coupling Branch Modal Models, Int. J. Thermal Sci., vol. 48, pp. 1060-1067, 2009.

18. C. Lanczos, An Iteration Method for the Solution of the Eigenvalue Problem of Linear Differential and Integral Operators, J. Res. Natl. Bur. Stand., vol. 45, pp. 225-280, 1950.

19. Y. Saad, Numerical Methods for Large Eigenvalue Problems, in Classics in Applied Mathematics, John Wiley \& Sons, 2011.

20. R. Lehoucq and D. Sorenson, Deflation Technics, for an Implicitly Restarted Arnoldi Iteration, CAAM-TR 94-13, Rice University Houston, TX, 1994.

21. R. Lehoucq, D. Sorenson, and C. Yang, Arpack User's Guide Soluiton of Large Scale Eigenvalue Problem with Implicit Restarted Arnoldi Method. SIAM, Philadelphia, 1998.

22. M. Girault, E. Videcoq, and D. Petit, Estimation of Time-Varying Heat Sources through Inversion of a Low Order Model Built with the Modal Identification Method from in-Situ Temperature Measurements, Int. J. Heat Mass Transfer, vol. 53, pp. 206-219, 2010. 


\section{APPENDIX: DISCRETE FORMULATION}

This appendix presents the matrix relations corresponding to the different equations of this article. They are obtained from spatial discretization by classical finite-element method with linear shape functions, giving $N_{\text {mesh }}$ nodes. The following equations are obtained by respecting the appearance order of the different terms in the analytical relations. These discrete formulations permit us to highlight the dimensions of the different relations.

\begin{tabular}{|c|c|c|c|}
\hline & Global technique & & Substructuring technique \\
\hline $\begin{array}{l}\text { Heat } \\
\text { equation }\end{array}$ & $\mathbf{C}_{\Omega} \frac{d \mathbf{T}}{d t}=(\mathbf{K}+\mathbf{A}) \mathbf{T}+\Pi_{\Omega}+\Pi_{\Gamma}$ & Eq. (5) & $\begin{aligned} \mathbf{C}_{\Omega} \frac{d \mathbf{T}}{d t} & =(\mathbf{K}+\mathbf{A}) \mathbf{T} \\
& +\Pi_{\Omega}+\Pi_{\Gamma}+\mathbf{J}_{\mathbf{T}} \mathbf{T}\end{aligned}$ \\
\hline $\begin{array}{l}\text { Eigenvalues } \\
\text { problem }\end{array}$ & $\mathbf{K}_{0} \mathbf{V}_{i}=-z_{i}\left(\mathbf{C}_{\Omega 0}+\mathbf{C}_{\Gamma}\right) \mathbf{V}_{i}$ & Eq. (10) & $\begin{array}{l}\mathbf{K}_{0} \mathbf{V}_{i}^{(1)}=-z_{i}^{(1)}\left(\mathbf{C}_{\Omega 0}+\mathbf{C}_{\Gamma}\right) \mathbf{V}_{i}^{(1)} \\
\mathbf{K}_{0} \mathbf{V}_{i}^{(2)}=-z_{i}^{(2)}\left(\mathbf{C}_{\Omega 0}+\mathbf{C}_{\Gamma}\right) \mathbf{V}_{i}^{(2)} \quad \text { Eq. (24) } \\
\mathbf{K}_{0} \mathbf{V}_{i}^{(2)}=-z_{i}^{(3)}\left(\mathbf{C}_{\Omega 0}+\mathbf{C}_{\Gamma}\right) \mathbf{V}_{i}^{(3)}\end{array}$ \\
\hline Reduction & $\mathbf{V} \Rightarrow \widetilde{\mathbf{V}}$ & & $\left.\begin{array}{rl}\mathbf{V}^{(1)} & \Rightarrow \widetilde{\mathbf{V}}^{(1)} \\
\mathbf{V}^{(2)} & \Rightarrow \widetilde{\mathbf{V}}^{(2)} \\
\mathbf{V}^{(3)} & \Rightarrow \widetilde{\mathbf{V}}^{(3)}\end{array}\right\} \Rightarrow \widehat{\mathbf{V}}$ \\
\hline \multirow[t]{2}{*}{$\begin{array}{l}\text { States } \\
\text { equation }\end{array}$} & \multirow{2}{*}{\multicolumn{2}{|c|}{$\begin{aligned} \widetilde{\mathbf{V}}^{\mathrm{t}} \mathbf{C}_{\Omega} \widetilde{\mathbf{V}} \frac{d \mathbf{X}}{d t} & =\widetilde{\mathbf{V}}^{\mathbf{t}}(\mathbf{K}+\mathbf{A}) \widetilde{\mathbf{V}} \mathbf{X} \\
& +\widetilde{\mathbf{V}}^{\mathbf{t}}\left(\Pi_{\Omega}+\Pi_{\Gamma}\right) \text { Eq. }\end{aligned}$}} & $\begin{aligned} \widehat{\mathbf{V}}^{\mathbf{t}} \mathbf{C}_{\Omega} \widehat{\mathbf{V}} \frac{d \mathbf{X}}{d t} & =\widehat{\mathbf{V}}^{\mathbf{t}}(\mathbf{K}+\mathbf{A}) \widehat{\mathbf{V}} \mathbf{X} \\
& +\widehat{\mathbf{V}}^{\mathbf{t}}\left(\Pi_{\Omega}+\Pi_{\Gamma}\right)\end{aligned}$ \\
\hline & & & $+\widehat{\mathbf{V}}^{\mathbf{t}} \mathbf{J}_{\mathbf{T}} \widehat{\mathbf{V}} \mathbf{X}$ \\
\hline
\end{tabular}

The different matrices are as follows.

1. Concerning the heat equation, $\mathbf{T}$ is the unknown temperature vector. $\mathbf{C}_{\Omega}$ and $\mathbf{K}$ are respectively the capacity matrix and the conductance matrix. $\mathbf{A}$ is the matrix of exchange through the external boundary $\Gamma e$. The vectors $\Pi_{\Omega}$ et $\Pi_{\Gamma}$ correspond to the volume and surface (through $\Gamma e$ ) solicitations. All these matrices and vectors can depend on time and temperature. The matrix $\mathbf{J}_{\mathbf{T}}$ defines the flux coupling between subdomains through the contiguous boundaries $\Gamma c$.

In the classical global technique, the unknown number is $N_{\text {mesh }}$. For the substructuring technique, the dimension of this equation takes into account the double value of temperature at the $N_{\Gamma c}$ nodes which define the contiguous boundaries Gammac. The number of unknown is then $N_{\mathrm{STR}}=N_{\text {mesh }}+N_{\Gamma c}$.

2. In the eigenvalues problem, $\mathbf{V}_{i}$ is the discretized eigenvector $i$ associated to the eigenvalue $z_{i}$, the size of which corresponds to the number of nodes used for the domain (or subdomain) considered $N_{\text {mesh }}$. The matrices $\mathbf{K}_{0}, \mathbf{C}_{\Omega 0}$, and $\mathbf{C}_{\Gamma}$ are, respectively, the conductance matrix, the capacity matrix, and the Steklov matrix, which correspond to fixed values of parameters. Because a truncation is performed when the Lanczos method is used, the number of eigenvectors is $N_{0}<N_{\text {mesh }}$. 
3. In the reduction step, the matrix $\mathbf{V}\left[N_{\text {mesh }}, N_{0}\right]$ contains all the $N_{0}$ eigenvectors $\mathbf{V}_{\mathbf{i}}$. The reduction leads to a matrix $\widetilde{\mathbf{V}}\left[N_{\text {mesh }}, \widetilde{N}\right]$. In the substructuring technique, $\widehat{\mathbf{V}}\left[N_{\mathrm{STR}}, \widehat{N}\right]$, is built (with $\widehat{N}=\widetilde{N}^{(1)}+\widetilde{N}^{(2)}+\widetilde{N}^{(3)}$ ), by adding each substructuring reduced basis, which are extended by zero on the other subdomains.

4. For the state equation, the dimension of such matrix formulation is then equal to $[\widetilde{N}, \widetilde{N}]$ for the global method, and to $[\widehat{N} \widehat{N}]$ for the substructuring method. These dimensions can be compared with the dimension of the classical finite-element formulation, described by Eq. (5). 